\title{
Avaliação do Metabolismo da Glicose em Pacientes Acromegálicos Antes e Após Tratamento com Octreotide LAR
}

\section{RESUMO}

\begin{abstract}
Objetivo: Avaliar o metabolismo da glicose em pacientes acromegálicos antes e após o tratamento com octreotide LAR. Pacientes e métodos: Este foi um estudo longitudinal e prospectivo com 30 pacientes do ambulatório de pesquisa em acromegalia do Serviço de Endocrinologia do Hospital Universitário Clementino Fraga Filho da Universidade Federal do Rio de Janeiro (HUCFF/UFRJ). Eles foram submetidos à avaliação clínica e laboratorial com dosagens de hormônio do crescimento $(\mathrm{GH})$, fator de crescimento semeIhante à insulina tipo I (IGF-I), insulina, pró-insulina, peptídeo $C$, hemoglobina glicosilada (HbA1c), proteína de ligação do IGF tipo 1 (IGFBP-1) e a um teste oral de tolerância à glicose (TOTG), antes e após seis meses de tratamento com octreotide LAR. Foi aplicado o teste dos postos sinalizados de Wilcoxon e o critério de determinação de significância adotado foi o nível de $5 \%$. Resultados: Encontraram-se 16 pacientes (54\%) com tolerância normal à glicose, sete $(23 \%)$ com intolerância à glicose e sete (23\%) com diabetes melito (DM). Doze pacientes completaram os seis meses de tratamento, sendo que houve piora da tolerância à glicose em três e piora do controle glicêmico dos dois pacientes diabéticos. Houve aumento da circunferência abdominal $(p=0,03)$ e queda do GH $(p=0,04)$, \% IGF-I acima do limite superior do valor de referência (\%LSVR) $(p=0,001)$, insulina $(p=0,019)$, peptídeo $C(p=0,002)$ e do modelo de avaliação homeostática (HOMA-IR) $(p=0,039)$. Conclusões: Nesta série, o tratamento com octreotide LAR acarretou piora da tolerância à glicose em três pacientes não-diabéticos e piora do controle glicêmico em dois diabéticos, apesar da diminuição da resistência insulínica (RI). (Arq Bras Endocrinol Metab 2008;52/1:55-64)
\end{abstract}

Descritores: Acromegalia; Octreotide LAR; Metabolismo da glicose

\begin{abstract}
Evaluation of Glucose Metabolism in Acromegalic Patients Before and After Treatment with Octreotide LAR.

Aim of the study: To evaluate the glucose metabolism in acromegalic patients before and after treatment with octreotide LAR. Patients and Methods: This was a prospective and longitudinal study involving 30 patients from the acromegaly research outpatient clinic of the Endocrinology unit of the HUCFF/ UFRJ. They underwent clinical and laboratorial evaluations, with measurements of growth hormone $(\mathrm{GH})$, insulin-like growth factor type I (IGF-I), insulin, proinsulin, C peptide, glycosylated hemoglobin ( $\mathrm{HbA} 1 \mathrm{c})$, IGF binding protein type 1 (IGFBP-1) and glucose, during oral glucose tolerance test (OGTT), before and after six months of treatment with octreotide LAR. The Wilcoxon signed-rank test was used and values of $5 \%$ were considered statistically significant. Results: We found 16 (54\%) patients with normal glucose tolerance, $7(23 \%)$ with impaired glucose tolerance and $7(23 \%)$ diabetics. Twelve patients completed the six-month treatment, out of which three
\end{abstract}

artigo original

\author{
Lívia L. CoRrea \\ GISELLE F. TABOADA \\ FLÁVIA R. VAN HAUTE \\ Alessandra F. CASINI \\ Giovanna A. BalarinI \\ Leonardo Vieira Neto \\ EVELYN DE O. MACHADO \\ ROSITA FONTES \\ Cláudia C. de Andrade \\ Yolanda SCHRANK \\ MÔNICA R. GADELHA
}

Serviço de Endocrinologia, Hospital Universitário Clementino Fraga Filho, Universidade Federal do Rio de Janeiro, RJ, Brasil (LLC, GFT, FRVH, AFC, GAB, LVN, EOM, MRG); Instituto Estadual de Diabetes e Endocrinologia (IEDE), Rio de Janeiro, RJ, Brasil (LLC, GAB, RF, YS, MRG);

Laboratório Diagnósticos da América, Rio de Janeiro, RJ, Brasil (RF, CCA, YS)

Recebido em 18/07/2007 Aceito em 30/10/2007 
showed worsening of glucose tolerance and two (diabetics) had worse blood glucose control. Whereas there was an increase in waist circumference $(p=0.03)$, there was a decrease in $\mathrm{GH}(p=0.04)$, with \%IGF-I above the upper limit of reference values ( $\%$ ULRV) $[p=0.001]$, insulin ( $p=0.019), C$ peptide levels $(p=0.002)$ and homeostatic model assessment (HOMA-IR) [ $p=0.039]$. Conclusions: In this series, treatment with octreotide LAR led to a worsening of glucose tolerance in three non-diabetic patients and worsened glycemic control in two diabetics, in spite of reducing insulin resistance. (Arq Bras Endocrinol Metab 2008;52/1:55-64)

Keywords: Acromegaly; Octreotide LAR; Glucose metabolism

\section{INTRODUÇÃO}

$\mathrm{O}$ MECANISMO ENVOLVENDO a resistência insulínica (RI) na acromegalia ainda é pouco compreendido. Já foi proposto que a RI ocorra ao nível do receptor de insulina (1). De fato, a diminuição da concentração de receptores e de sua afinidade pela insulina foi descrita, entretanto, o defeito induzido pelo hormônio do crescimento (growth hormone - GH) parece envolver também um sítio pós-receptor, como demonstrado em modelos animais (2). Um estudo com pacientes acromegálicos mostrou uma ligação normal da insulina a monócitos e eritrócitos, reforçando a hipótese de um defeito predominantemente pós-receptor (3). Esses defeitos causam redução da supressão da produção hepática de glicose e prejuízo em seu transporte para tecidos periféricos (1). Um mecanismo adicional envolvido na RI é o excesso de ácidos graxos livres circulantes resultantes da ação lipolítica do GH. O aumento dos níveis de glicose e ácidos graxos livres serve como estímulo à secreção de insulina, causando hiperinsulinemia sustentada (4).

A administração do fator de crescimento semelhante à insulina tipo I (insulin-like growth factor type IIGF-I) tanto em animais como em seres humanos cursa com aumento da sensibilidade à insulina, contrastando com os efeitos do $\mathrm{GH}(5,6)$. Acredita-se que essa ação seja mediada por meio da musculatura esquelética, pois a diminuição dos níveis de IGF-I é acompanhada do aumento da RI, principalmente em tecido muscular (7). Desta forma, o GH tem um efeito direto, induzindo a RI, e um indireto, por intermédio do IGF-I, aumentando a sensibilidade à insulina. No entanto, o efeito do IGF-I não consegue impedir o desenvolvimento da RI característico da acromegalia (8).

Se a RI for maior que a capacidade de produção compensatória de insulina, ocorrerá intolerância à glicose, presente em cerca de $50 \%$ dos casos ou diabetes melito (DM), presente em $10 \%$ a $25 \%$ (9). Hiperinsulinemia, intolerância à glicose e DM estão associados com o aumento da mortalidade cardiovascular, responsável por cerca de $60 \%$ dos óbitos em acromegálicos (10-12).

Outros marcadores bioquímicos de alteração do metabolismo glicídico estão presentes na acromegalia. Além da hiperinsulinemia, pacientes com acromegalia costumam ter níveis séricos de pró-insulina e peptídeo C mais elevados que controles normais $(13,14)$, sendo o aumento dos níveis circulantes de pró-insulina considerado um reflexo da disfunção da célula-beta pancreática (14). Por outro lado, os níveis da proteína de ligação do IGF-I tipo 1 (insulin like growth factor binding protein type 1- IGFBP-1) tendem a ser baixos, visto sua regulação ser dependente da insulina, evidenciando sua relação inversa com a hiperinsulinemia existente (15-17).

Os análogos da somatostatina representam o tratamento medicamentoso de escolha da acromegalia, entretanto, o metabolismo glicídico pode ser afetado por essa terapia. O octreotide pode causar aumento da concentração sangüínea de glicose por meio da inibição da secreção de insulina pelas células-beta pancreáticas $(13,18)$. Além disso, os análogos da somatostatina aumentam a concentração de IGFBP-1 tanto por conseqüência da diminuição da secreção de insulina (15) quanto por indução direta (16). Embora o IGFBP-1 
possa facilitar algumas ações celulares do IGF-I in vitro (19), essa proteína tem efeito predominantemente inibitório sobre as ações do IGF-I (20). De fato, o aumento dos níveis de IGFBP-1 reduz o efeito insulínico do IGF-I com conseqüente aumento da glicemia (16).

Por outro lado, o octreotide pode causar redução dos níveis de glicose pela inibição da hipersecreção de GH. A redução dos níveis circulantes de $\mathrm{GH}$ leva à inibição da gliconeogênese e ao aumento da sensibilidade à insulina, com melhora de sua ação tanto ao nível do receptor como no mecanismo pós-receptor $(13,18)$. Além disso, o octreotide inibe a secreção de glucagon e outros hormônios gastrintestinais, como gastrina e motilina, reduzindo a motilidade gastrintestinal com conseqüente diminuição da absorção de glicose $(21,22)$. Finalmente, há redução da depuração de insulina que também contribui para a queda da glicemia (23).

Esses efeitos divergentes podem explicar as conseqüências imprevisíveis do octreotide no metabolismo da glicose. O presente estudo se propôs a estudar o metabolismo da glicose em uma população de acromegálicos, antes e após seis meses de tratamento com o octreotide LAR. Foram realizados o teste oral de tolerância à glicose (TOTG) e as dosagens de insulina, peptídeo C, pró-insulina, hemoglobina glicosilada (HbAlc) e IGFBP-1.

\section{PACIENTES E MÉTODOS}

\section{Pacientes}

O presente estudo foi aprovado pelo Comitê de Ética e Pesquisa do HUCFF/UFRJ. Todos os pacientes foram esclarecidos em relação aos objetivos do trabalho e dos procedimentos a que seriam submetidos e assinaram o termo de consentimento livre e esclarecido.

Foram selecionados, ao longo de 12 meses, $30 \mathrm{pa}$ cientes do ambulatório de pesquisa em acromegalia do Serviço de Endocrinologia do HUCFF/UFRJ, sendo $16(53,3 \%)$ do sexo feminino. Em $73,4 \%$ dos pacientes $(22 / 30)$ o tumor era um macroadenoma, em 13,3\% (4/30), um microadenoma e 13,3\% (4/30) apresentavam sela vazia à época da inclusão no estudo. Quatorze $(46,7 \%)$ já haviam sido submetidos ao tratamento cirúrgico, e dois $(6,7 \%)$ haviam recebido tratamento radioterápico adjuvante à cirurgia. Oito $(26,7 \%)$ pacientes já haviam recebido tratamento medicamentoso, sendo três deles com bromocriptina somente, dois com bromocriptina e octreotide subcutâneo (SC), dois com bromocriptina e cabergolina e um com apenas cabergo- lina. Destes oito pacientes, quatro já haviam feito cirurgia e dois cirurgia e radioterapia, que havia sido concluída há 14 e oito anos, respectivamente. Os dois restantes usaram medicações como tratamento primário da acromegalia. Quatorze $(46,7 \%)$ nunca haviam recebido nenhum tratamento. Apesar do tratamento prévio, todos os pacientes apresentavam evidências clínicas e laboratoriais de atividade da doença. Dos 30 pacientes analisados, $14(46,7 \%)$ não apresentavam déficit de trofinas associado, sete $(23,3 \%)$ apresentavam deficiência do setor tireotrófico, sete $(23,3 \%)$ do setor corticotrófico e 11 (36,7\%) do setor gonadotrófico. Dentre os pacientes com déficit de hormônios tireoidianos e de cortisol, todos faziam reposição com levotiroxina e prednisona, respectivamente, apresentando-se controlados à época da avaliação. Dois pacientes com hipogonadismo central faziam reposição de testosterona.

Foram excluídos do estudo acromegálicos com controle da doença, que já tivessem feito uso prévio de octreotide LAR (long-acting repeatable), que tivessem feito uso de octreotide SC e/ou agonistas dopaminérgicos há menos de seis meses do início do octreotide LAR, portadores de DM secundário a outras causas e gestantes.

\section{MÉTODOS}

As definições de DM, intolerância à glicose e glicemia de jejum alterada tem como base o último consenso da American Diabetes Association (ADA) (24). A RI foi avaliada pelo modelo de avaliação homeostática (bomeostatic model assessment - HOMA-IR) (25).

O TOTG não foi realizado nos pacientes sabidamente diabéticos, bem como as dosagens de insulina, pró-insulina, peptídeo C e IGFBP-1 nos diabéticos em tratamento medicamentoso. Foi utilizada a porcentagem de incremento do IGF-I acima do limite superior do valor de referência (\%LSVR) para análise, já que o IGF-I apresenta distintos valores de referência nas diferentes décadas de vida.

As amostras foram coletadas pela manhã, estando o paciente em jejum de oito horas. O método utilizado para dosagem de GH foi quimioluminescência (kit comercial da DPC - Diagnostic Products Corporation, Los Angeles, CA, com o analisador IMMULITE ${ }^{\circledast} 1000$ ), direcionado especificamente para detecção da isoforma de GH com peso molecular de $22 \mathrm{KDa}$. A sensibilidade do método é de $0,01 \mathrm{ng} / \mathrm{mL}$ com coeficientes de variação inter e intra-ensaio de 6,2\% e 6,5\%, respectivamente. 
O método imunorradiométrico com extração prévia das proteínas carreadoras com etanol (kit comercial da DSL - Diagnostic Systems Labaratories, Inc., Webster, TX) foi utilizado para a dosagem de IGF-I. A sensibilidade do método é de $0,80 \mathrm{ng} / \mathrm{mL}$. O coeficiente de variação interensaio é de $8,2 \%, 1,5 \%$ e $3,7 \%$ para baixo, médio e alto ponto da curva-padrão, respectivamente. O coeficiente de variação intra-ensaio é de $3,4 \%$, $3,0 \%$ e 1,55 para baixo, médio e alto ponto da curvapadrão, respectivamente. Os valores de referência (VR), em $\mathrm{ng} / \mathrm{mL}$, variam conforme a idade e o sexo do paciente, sendo de 18 a 20 anos: 197-956 para homens e 193-575 para mulheres; 20 a 23 anos: 215-628 e 110-521; 23 a 25 anos: 169-591 e 129-480; 25 a 30 anos: 119-476 e 96-502; 30 a 40 anos: 100-494 e 130-354; 40 a 50 anos: 101-303 e 50-70 anos: 78-258. Após os 40 anos, os VRs utilizados são os mesmos para ambos os sexos.

Para glicose, o método foi cinético UV hexoquinase com o analisador Dimension RXL Dade Behring. A sensibilidade do método é de $0,80 \mathrm{mg} / \mathrm{dL}$, com coeficientes de variação inter e intra-ensaio de 1,4\% e 2,82\%, respectivamente, e com VR de 70-99 $\mathrm{mg} / \mathrm{dL}$.

Foi feita a dosagem de insulina, utilizando quimioluminescência (kit comercial da DPC com o analisador IMMULITE $\left.^{\circledR} 2000\right)$. A sensibilidade do método é de 2 $\mathrm{mcUI} / \mathrm{mL}$ com coeficientes de variação inter e intraensaio de 4,9\% e 3,9\%, respectivamente, e VR de 6-27 $\mathrm{mcUI} / \mathrm{mL}$.

Para peptídeo C, o método foi quimioluminescência (kit comercial da DPC com o analisador IMMULITE ${ }^{\circledR}$ 1000) e sensibilidade de $0,3 \mathrm{ng} / \mathrm{mL}$. O coeficiente de variação interensaio é $8,1 \%$ e intra-ensaio é $10 \%$. O VRé de 0,9-4,0 ng/mL.

O método utilizado para dosagem de HbAlc foi HPLC (kit comercial da Bio-Rad Laboratories, Hercules, CA, com o analisador VARIANT II) e sensibilidade de $1,3 \%$. Os coeficientes de variação inter e intra-ensaio são de $1,84 \%$ e 1,46\%, respectivamente, e com VR de $4,1 \%$ a $6,5 \%$.

O método para dosagem de pró-insulina foi imunorradiométrico (Human Proinsulin RIA Kit, Saint Charles, Missouri, com o analisador Perkin Elmer). A sensibilidade do método é de 2 pMol quando o ensaio é realizado usando um volume de amostra de $200 \mathrm{mi}$ crolitros. Ambos os coeficientes de variação são de 1,5\% e o VR é de 6,4 a 9,4 pMol/mL.

Finalmente, a dosagem de IGFBP-1 foi realizada pelo laboratório Diagnósticos da América Medicina
Diagnóstica, no laboratório Quest Diagnostics, nos Estados Unidos. O método foi o imunorradiométrico, utilizando um equipamento in house, com sensibilidade de $0,5 \mathrm{ng} / \mathrm{mL}$ e coeficiente de variação intra-ensaio cumulativo de $4,6 \%$ a $10 \%$. O VR é de 13 a $73 \mathrm{ng} / \mathrm{mL}$.

Foram feitas também avaliações do índice de massa corporal (IMC), por meio das medidas de peso $(\mathrm{kg}) \mathrm{e}$ altura $(\mathrm{m})$ e definido como peso sobre a altura ao quadrado e da circunferência abdominal (CA), medida em centímetros na metade da distância entre a espinha ilíaca ântero-superior e a borda inferior do último arco costal, antes e após seis meses de tratamento, por um único examinador (26).

Todos os pacientes iniciaram o tratamento com octreotide LAR $20 \mathrm{mg}$ a cada 28 dias. Após três meses de uso da medicação, o eixo do GH foi testado. Se o IGF-I permanecesse elevado para idade e sexo e/ou os níveis de $\mathrm{GH}$ estivessem acima de $2,5 \mathrm{ng} / \mathrm{mL}$, a dose era aumentada para $30 \mathrm{mg}$.

\section{Análise Estatística}

Em uma abordagem exploratória dos dados, foi proposto analisar a variação das medidas clínicas e laboratoriais após seis meses de tratamento, aplicando o teste dos postos sinalizados de Wilcoxon.

O critério de determinação de significância adotado foi o nível de 5\%. A análise estatística foi processada pelo software estatístico $\mathrm{SAS}^{\circledR}$ System, versão 6.04, Cary, NC, USA.

\section{RESULTADOS}

Dos 30 pacientes estudados, 16 (54\%) apresentavam tolerância normal à glicose, sete $(23 \%)$ intolerância à glicose ou glicemia de jejum alterada e sete $(23 \%)$ eram diabéticos.

A tabela 1 fornece as características clínicas e laboratoriais dos 30 pacientes em estudo. Foram feitas dosagens de insulina, pró-insulina, IGFBP-1 e cálculo do HOMA-IR de 24 pacientes, pois seis eram diabéticos em tratamento medicamentoso. Uma paciente diabética não tinha dados antropométricos completos por ter sido previamente submetida à amputação suprapatelar bilateralmente. Houve falha na coleta da HbAlc e peptídeo C dessa mesma paciente.

Doze pacientes foram avaliados após seis meses de tratamento com octreotide LAR. As características clínicas e laboratoriais desses pacientes antes e após seis meses de tratamento podem ser vistas na tabela 2 . 
Tabela 1. Análise descritiva das variáveis numéricas basais para amostra geral

\begin{tabular}{lcccc}
\hline Variável & $\mathbf{n}$ & Mediana & Mínimo & Máximo \\
\hline Idade (anos) & 30 & 45,5 & 23 & 75 \\
\hline TD (meses) & 30 & 60 & 12 & 300 \\
\hline \%LSVR & 30 & 149 & -32 & 571 \\
\hline GH (ng/mL) & 30 & 13,3 & 1,4 & 198 \\
\hline Glicose $(\mathrm{mg} / \mathrm{dL})$ & 30 & 97,5 & 79 & 429 \\
\hline Insulina $(\mathrm{mcUl} / \mathrm{mL})$ & 24 & 11 & 6,24 & 47,9 \\
\hline Pró-insulina (pMol/mL) & 24 & 12 & 4,8 & 34,1 \\
\hline Peptídeo C $(\mathrm{ng} / \mathrm{mL})$ & 23 & 2,3 & 1 & 7 \\
\hline IGFBP-1 $(\mathrm{ng} / \mathrm{mL})$ & 24 & 11,5 & 5 & 87 \\
\hline HbAlc $(\%)$ & 29 & 5,7 & 5,1 & 14,4 \\
\hline HOMA-IR & 24 & 2,3 & 1,4 & 10,9 \\
\hline CA (cm) & 29 & 100 & 77 & 128 \\
\hline IMC (kg/m²) & 29 & 29,3 & 22,6 & 50,8 \\
\hline
\end{tabular}

$T D=$ tempo de doença; \%LSVR= porcentagem de incremento do IGF-I acima do limite superior do valor de referência; $\mathrm{GH}=$ hormônio do crescimento; IGFBP-1= proteína de ligação do IGF tipo 1; HbAlc= hemoglobina glicosilada; HOMA-IR= modelo de avaliação homeostática-resistência insulínica; CA-circunferência abdominal; IMC= índice de massa corporal.

Tabela 2. Características clínicas e laboratoriais dos pacientes antes e após seis meses de tratamento com octreotide LAR.

\begin{tabular}{|c|c|c|c|c|c|c|c|c|c|c|c|c|c|c|c|c|c|c|c|c|c|c|c|c|c|c|}
\hline \multirow{2}{*}{$\begin{array}{c}\text { IMC } \\
\mathrm{N}\end{array}$} & \multicolumn{2}{|c|}{ IMC } & \multicolumn{2}{|c|}{ CA } & \multicolumn{2}{|c|}{ GIl bas } & \multicolumn{2}{|c|}{ Gllc $2 h$} & \multicolumn{2}{|c|}{$\mathrm{HbAlc}$} & \multicolumn{2}{|c|}{ GH } & \multicolumn{2}{|c|}{ IGF-I } & \multicolumn{2}{|c|}{ Insulina } & \multicolumn{2}{|c|}{ Pró-Insul } & \multicolumn{2}{|c|}{ Pep. C } & \multicolumn{2}{|c|}{ IGFBP-1 } & \multicolumn{2}{|c|}{ HOMA } & \multicolumn{2}{|c|}{ LAR } \\
\hline & B & 6 & B & 6 & B & 6 & B & 6 & B & 6 & B & 6 & B & 6 & B & 6 & B & 6 & B & 6 & B & 6 & B & 6 & B & 6 \\
\hline 1 & 32,4 & 33,6 & 92 & 95 & 97 & 96 & 107 & 122 & 6,0 & 6,1 & 12,6 & 3,4 & 2376 & 516 & 46 & 7,6 & 17,1 & 10,6 & 7 & 1,9 & 6 & 21 & 11 & 1,8 & 20 & 30 \\
\hline 2 & 31,1 & 31,1 & 107 & 112 & 98 & 83 & 126 & 160 & 6,1 & 6,5 & 2,7 & 0,7 & 752 & 277 & 10 & 3,4 & 6 & 6,4 & 1,4 & 0,9 & 9 & 14,8 & 2,4 & 0,7 & 20 & 20 \\
\hline 3 & 27,6 & 27,5 & 85 & 86 & 82 & 91 & 75 & 73 & 5,6 & 5,6 & 10,1 & 3,9 & 625 & 404 & 9,5 & 7,2 & 33,7 & 16,9 & 3,2 & 1,1 & $<5$ & 10 & 1,9 & 1,6 & 20 & 30 \\
\hline 4 & 24,5 & 23,8 & 83 & 81 & 82 & 86 & 89 & 151 & 5,4 & 5,6 & 46,1 & 72,3 & 875 & 639 & 11,5 & 11 & 5,4 & 11,6 & 2,1 & 1,2 & 12,4 & 16,7 & 2,3 & 2,3 & 20 & 30 \\
\hline 5 & 24,2 & 25,9 & 86 & 86 & 87 & 91 & 62 & 108 & 5,5 & 5,4 & 17 & 24,2 & 1864 & 579 & 15,2 & 7,9 & 11,6 & 12,7 & 2 & 1,2 & $<5$ & 29,3 & 3,3 & 1,8 & 20 & 30 \\
\hline 6 & 34,7 & 36,5 & 107 & 106 & 80 & 88 & 90 & 128 & 5,7 & 5,7 & 197 & 143 & 1178 & 1382 & 6,2 & 12,5 & 8,6 & 15,7 & 1,8 & 1,1 & 11,5 & 11 & 1,2 & 2,7 & 20 & 30 \\
\hline 7 & 29,7 & 30,5 & 91 & 93 & 99 & 116 & 103 & 117 & 5,6 & 6 & 7,1 & 1,7 & 1124 & 224 & 20,8 & 6,2 & 8,1 & 10,3 & 3 & 1,7 & 31,6 & 22,3 & 5,1 & 1,8 & 20 & 20 \\
\hline 8 & 29,6 & 33,9 & 100 & 109 & 82 & 79 & 95 & 132 & 6,4 & 5,8 & 32,7 & 0,1 & 327 & 175 & 10,1 & 6,4 & 23,5 & 17,6 & 4,4 & 2,5 & 11,9 & 10,9 & 2 & 1,2 & 20 & 20 \\
\hline 9 & 27,9 & 28,1 & 100 & 103 & 99 & 83 & 191 & 185 & 5,3 & 5,3 & 3,8 & 0,2 & 268 & 97 & 6,6 & 3,8 & 11,2 & 6,4 & 1,5 & 0,8 & 23,6 & 30,6 & 1,6 & 0,8 & 20 & 20 \\
\hline 10 & 30,9 & 30,9 & 102 & 109 & 115 & 117 & 102 & 89 & 6,1 & 5,7 & 30,2 & 1,7 & 1855 & 553 & 41,3 & 2 & 15,3 & 7,7 & 6,4 & 1,4 & $<5$ & 20,3 & 11,7 & 0,6 & 20 & 30 \\
\hline 11 & 29,3 & 28,9 & 96 & 95 & 103 & 109 & - & - & 5,9 & 6,3 & 5,6 & 0,5 & 858 & 376 & - & - & - & - & - & - & - & & - & - & 20 & 30 \\
\hline 12 & 25,7 & 25,4 & 85 & 89 & 123 & 128 & - & - & 6,5 & 6,6 & 6,5 & 3,8 & 1.241 & 250 & & & & & & & & & & - & 20 & 30 \\
\hline
\end{tabular}

$\mathrm{N}=$ número; $\mathrm{B}=$ basal; $6=6$ meses; $\mathrm{IMC}=$ índice de massa corporal; $\mathrm{CA}=$ circunferência abdominal; Gli bas= glicose basal; Gli $2 \mathrm{~h}$ = glicose de 2 horas; $\mathrm{HbAlc}=$ hemoglobina glicosilada; $\mathrm{GH}=$ hormônio do crescimento; $\mid \mathrm{GF}-\mathrm{I}=$ fator de crescimento semelhante à insulina tipo l; Pró-insul= pró-insulina; Pep C= peptídeo C; IGFBP-1= proteína de ligação do IGF tipo 1; HOMA= modelo de avaliação homeostática= resistência insulínica; LAR= dose em mg de octreotide LAR. Algumas avaliações não foram feitas nos pacientes 11 e 12 por serem diabéticos. 
A tabela 3 fornece a mediana, o mínimo e o máximo da variação absoluta (sexto mês - basal) das medidas laboratoriais e clínicas e o correspondente nível descritivo ( $p$ valor) do teste estatístico para todos os 12 pacientes, independentemente da tolerância à glicose. A tabela 4 fornece os mesmos valores para dez pacientes, excluindo os dois diabéticos identificados no início do tratamento.

No grupo, como um todo, houve queda significativa após seis meses de tratamento nas medidas de $\% \operatorname{LSVR}(\mathrm{p}=0,001)$, tendo passado de uma mediana no basal de 212 para 23 após seis meses de tratamento, GH $(\mathrm{p}=0,04)$, com mediana diminuindo de 14,8 para 2,45 , respectivamente, no basal e com seis meses e, finalmente, aumento significativo na circunferência abdominal $(\mathrm{p}=$ 0,03), com valor da mediana passando de 94 para 95. As análises da insulina, pró-insulina, peptídeo C, IGFBP-1 e HOMA-IR não foram feitas, pois existiam dois pacientes diabéticos em uso de metformina.

Excluídos os dois pacientes diabéticos, observou-se queda significativa após seis meses de tratamento nas medidas de \%LSVR ( $\mathrm{p}=0,003)$, com diminuição da

Tabela 3. Análise estatística da variação absoluta ( $6^{\circ}$ mês - basal) para todos os 12 pacientes.

\begin{tabular}{lccccc}
\hline Variação (6m-basal) & $\mathbf{n}$ & Mediana & Mínimo & Máximo & p valor \\
\%LSVR & 12 & -186 & -526 & 41 & $\mathbf{0 , 0 0 1}$ \\
GH (ng/mL) & 12 & $-6,15$ & $-61,7$ & 26,1 & $\mathbf{0 , 0 4 0}$ \\
Glicose (mg/dL) & 12 & 4 & -16 & 24 & 0,29 \\
HbAlc (\%) & 12 & 0,05 & $-0,6$ & 0,4 & 0,48 \\
CA (cm) & 12 & 2,5 & -2 & 9 & $\mathbf{0 , 0 3 0}$ \\
IMC $\left(\mathrm{kg} / \mathrm{m}^{2}\right)$ & 12 & 0,1 & $-0,7$ & 4,3 & 0,16 \\
\hline
\end{tabular}

\%LSVR= porcentagem de incremento do IGF-I acima do limite superior do valor de referência; $\mathrm{GH}=$ hormônio do crescimento; $\mathrm{HbAlc}=$ hemoglobina glicosilada; $\mathrm{CA}=$ circunferência abdominal; $\mathrm{IMC}=$ índice de massa corporal.

Tabela 4. Análise estatística da variação absoluta ( $6^{\circ}$ mês - basal) para 10 pacientes (excluídos os 2 diabéticos).

\begin{tabular}{lccccc}
\hline Variação (6m-basal) & $\mathbf{n}$ & Mediana & Mínimo & Máximo & p valor \\
\%LSVR & 10 & $-128,5$ & -526 & 41 & $\mathbf{0 , 0 0 3}$ \\
GH (ng/mL) & 10 & $-6,15$ & $-32,6$ & 26,1 & 0,10 \\
Glicose (ng/dL) & 10 & 3 & -16 & 9 & 0,68 \\
Insulina (mcUl/mL) & 10 & $-5,12$ & $-39,26$ & 6,26 & $\mathbf{0 , 0 1 9}$ \\
Pró-insulina (pMol/mL) & 10 & $-2,2$ & $-16,8$ & 7,1 & 0,49 \\
Peptídeo C (ng/mL) & 10 & $-1,1$ & $-5,1$ & $-0,5$ & $\mathbf{0 , 0 0 2}$ \\
IGFBP-1 (ng/mL) & 10 & 5,4 & $-9,3$ & 24,3 & 0,084 \\
HbAlc (\%) & 10 & 0 & $-0,6$ & 0,4 & 0,89 \\
HOMA- IR & 10 & $-1,15$ & $-11,1$ & 1,5 & $\mathbf{0 , 0 3 9}$ \\
CA (cm) & 10 & 2,5 & -2 & 9 & $\mathbf{0 , 0 3 9}$ \\
IMC (kg/m²) & 10 & 0,5 & $-0,7$ & 4,3 & 0,054 \\
\hline
\end{tabular}

\%LSVR= porcentagem de incremento do IGF-I acima do limite superior do valor de referência; GH= hormônio do crescimento; IGFBP-1= proteína de ligação do IGF tipo 1; HbAlc= hemoglobina glicosilada; HOMA-IR= modelo de avaliação homeostática - resistência insulínica; $C A=$ circunferência abdominal; $I M C=$ índice de massa corporal. 
mediana de 164,5 no basal para 23 após seis meses, insulina ( $\mathrm{p}=0,019)$, com medianas de, respectivamente, 10,8 e 6,8, no basal e após tratamento, peptídeo C ( $\mathrm{p}=0,002$ ), que passou de 2,55 para 1,2, HOMA-IR ( $\mathrm{p}$ $=0,039)$, com queda de 2,35 para 1,7 e aumento significativo na circunferência abdominal $(\mathrm{p}=0,039)$, com medianas evoluindo de 96 para 99 com o tratamento. Houve também tendência de aumento no IGFBP-1 $(\mathrm{p}=0,084)$ e no IMC $(\mathrm{p}=0,054)$.

A tabela 5 fornece a variação da tolerância à glicose nos 12 pacientes estudados.

\section{DISCUSSÃo}

A prevalência de alterações do metabolismo glicídico em nossa casuística foi $46 \%$, sendo $23 \%$ de intolerantes à glicose e glicemia de jejum alterada e $23 \%$ de diabéticos. Isto é concordante com outras séries nacionais e internacionais, cuja prevalência varia entre $23 \%$ a $69 \%$ (8,18,27-29).

Na casuística, o IMC da amostra como um todo no basal foi $29,3(22,6-50,8) \mathrm{kg} / \mathrm{m}^{2}$, ora, existem evidências de que um IMC > 30 está associado a risco aumen- tado para doenças cardiovasculares e DM (26). No entanto, o excesso de $\mathrm{GH}$, por seu efeito lipolítico, leva a alteração na composição corporal com diminuição de tecido gorduroso e aumento da massa magra (30). Portanto, o elevado IMC encontrado na presente casuística pode ocorrer em virtude de aumento do tecido muscular e não de aumento do tecido adiposo (31).

$\mathrm{Na}$ acromegalia, RI com hiperinsulinemia e hiperproinsulinemia são dados freqüentemente descritos na literatura (14,32-34). Concordante com isso, observam-se níveis aumentados de pró-insulina e HOMAIR antes do início do tratamento medicamentoso com octreotide LAR. As concentrações de IGFBP-1 se encontravam próximas ao limite inferior da referência visto que a insulina contribui para a redução da concentração sérica dessa proteína transportadora. Assim, podemos concluir que esses pacientes têm risco aumentado de obesidade, dislipidemia, intolerância à glicose e diabetes (35).

Após seis meses de tratamento com octreotide LAR, houve queda estatisticamente significativa do $\% \operatorname{LSVR}(\mathrm{p}=0,001)$ e do GH $(\mathrm{p}=0,04)$, indicando a efetividade do tratamento da acromegalia. Oito pacientes encerraram o estudo em uso de $30 \mathrm{mg}$ de octreotide

Tabela 5. Variação da tolerância à glicose antes e após seis meses de tratamento.

\begin{tabular}{cccc}
\hline Número & \multicolumn{2}{c}{ Tolerância à glicose } & Variação da tolerância à glicose \\
\hline 1 & basal & 6 meses & $\Leftrightarrow$ \\
2 & TN & TN & $\Downarrow$ \\
3 & TN & IOG & $\Leftrightarrow$ \\
\hline 4 & TN & TN & $\Downarrow$ \\
5 & TN & IOG & $\Leftrightarrow$ \\
6 & TN & TN & $\Leftrightarrow$ \\
7 & TN & TN & $\Downarrow$ \\
8 & TN & GJA & $\Leftrightarrow$ \\
9 & TN & TN & $\Leftrightarrow$ \\
10 & IOG & IOG & $\Leftrightarrow$ \\
11 & IOG & IOG & $\Leftrightarrow$ \\
12 & DM & DM & $\Leftrightarrow$ \\
\hline & DM & DM & $\Leftrightarrow$ \\
\hline
\end{tabular}

TN=tolerância normal à glicose; $\mathrm{IOG}=$ intolerância à glicose; $\mathrm{DM}=$ diabetes melito;

$G J A=$ glicemia de jejum alterada; $\Leftrightarrow=$ estável; $\downarrow=$ piora. 
LAR. Foi observado aumento significativo da CA no grupo como um todo $(\mathrm{p}=0,03)$ e no grupo dos dez pacientes sem diabetes $(\mathrm{p}=0,039)$, possivelmente em virtude de um aumento do tecido adiposo pela redução dos níveis de GH e IGF-I. De fato, alguns estudos têm demonstrado que com o tratamento não se observam grandes variações no IMC, contudo, ocorre mudança na composição corporal com aumento do tecido gorduroso e diminuição da massa magra como conseqüência da queda dos níveis de GH/IGF-I $(31,36)$.

Considerando o grupo de dez pacientes não-diabéticos, observou-se melhora da RI, avaliada pelo HOMAIR, em oito deles (Tabela 2 ). Todos os oito obtiveram melhora clínica e laboratorial com a medicação, sendo que seis não modificaram sua tolerância à glicose e dois (pacientes de número 2 e 7) pioraram. Nestes, possivelmente a inibição da secreção de insulina pela medicação foi maior que a melhora da RI. Os dois pacientes que não apresentaram diminuição da RI (pacientes de número 4 e 6) foram os mesmos que não melhoraram clínica e laboratorialmente com o octreotide LAR. Estes achados estão em concordância com outros estudos nos quais houve melhora da RI após seis meses de tratamento com octreotide LAR, em decorrência predominantemente da diminuição do $\mathrm{GH}(37,38)$. Além disso, a diminuição da insulina e do peptídeo C observada na presente casuística é um resultado esperado pela inibição de sua secreção secundária à administração do análogo da somatostatina (37-39). Esse efeito inibitório é contínuo e progressivo conforme demonstrou estudo utilizando octreotide LAR por 24 meses (37).

Com relação à evolução da tolerância à glicose, nos pacientes não-diabéticos, as glicemias se mantiveram estáveis em $70 \%$ dos pacientes $(7 / 10)$ e pioraram em $30 \%$ (3/10). Em dois deles houve evolução de tolerância normal à glicose para intolerância à glicose e em outro para glicemia de jejum alterada. Não houve melhora da tolerância em nenhum paciente (Tabela 5). Houve piora do controle glicêmico dos dois pacientes diabéticos, tendo como base a HbAlc. Ambos faziam uso de metformina (Tabela 2). Se esses pacientes fizessem uso de insulina, possivelmente teria havido melhora das glicemias com o tratamento, como já foi mostrado em alguns estudos $(40,41)$. Nessa situação, o efeito direto dos análogos na função das células-beta pancreáticas promovendo a inibição da liberação de insulina é irrelevante, visto que a administração exógena de insulina e a melhora da glicemia devem-se à redução da resistência insulínica me- diada pelo controle dos níveis de $\mathrm{GH}$ e pela inibição da liberação de glucagon $(31,42)$.

Esses resultados estão de acordo com dados da literatura que mostram deterioração das glicemias de pacientes acromegálicos tratados com análogos da somatostatina. Em pacientes com glicemias inicialmente normais foi descrita piora da tolerância à glicose em $20 \%$ a 48\% $(18,43,44)$ e evolução para DM em 29\% (18). Não parece haver correlação entre a dose do octreotide e a mudança no metabolismo da glicose. No entanto, foi demonstrado que os indivíduos que apresentaram níveis mais elevados de insulina antes do tratamento tiveram mais propensão a desenvolver DM (18).

Entretanto, há estudos, embora sejam séries menores, relatando efeitos benéficos do octreotide no metabolismo da glicose. Ho e cols. (42) mostraram normalização da glicemia de quatro dos cinco pacientes com intolerância à glicose. Outra experiência positiva foi relatada por Colao e cols. (45) que observaram a normalização das glicemias de três dos sete pacientes acromegálicos diabéticos. Em dois outros houve substituição da insulina por agentes hipoglicemiantes orais, e nos dois restantes houve diminuição das doses de insulina.

A resposta do metabolismo da glicose ao tratamento com octreotide é resultado de um balanço entre seus efeitos inibitórios sobre a secreção pancreática de insulina e glucagon e da melhora da RI pela diminuição do GH (37). Nesta série de pacientes, o tratamento medicamentoso da acromegalia com octreotide LAR acarretou piora da tolerância à glicose nos pacientes, apesar da diminuição da RI.

\section{AGRADECIMENTOS}

Ao laboratório Diagnósticos da América Medicina Diagnóstica, fundamental na execução deste trabalho.

À Rosângela Aparecida Martins Noé, bioestatística da comissão de investigação científica do HUCFF/ UFRJ, pela análise estatística do estudo.

À enfermeira Mariangélica de O. Silva e à técnica em enfermagem Françoise R. Lopes, pela coleta dos exames e aplicação da medicação.

\section{REFERÊNCIAS}

1. Muggeo M, Robert $\mathrm{S}$, Roth J, Kahn R. The insulin resistance in acromegaly: evidence for two alterations in the insulin recep- 
tor on circulating monocytes. J Clin Endocrinol Metab. 1979; 48:17-25.

2. Gama R, Teale JD, Wright J, Ferns G, Marks V. Hyperproinsulinaemia in acromegaly: evidence for abnormal pancreatic beta- cell function. Ann Clin Biochem. 1997; 34:627-31.

3. Hansen I, Tsalikian E, Beaufrere B, Gerich J, Haymond M, Rizza $R$. Insulin resistance in acromegaly: defects in both hepatic and extrahepatic insulin action. Am J Physiol. 1986; 250:269-73.

4. Randle P, Garland P, Hales C, Newsholme E. The glucose fattyacid cycle: its role in insulin sensitivity and the metabolic disturbances of diabetes mellitus. Lancet. 1963; 1:785-9.

5. Rosseti L, Frontoni S, Dimarchi R. Metabolic effects of IGF-I in diabetic rats. Diabetes. 1991; 40:444-8.

6. Boulware SD, Tamborlane WV, Rennet NJ, Gesundheit N Sherwin RS. Comparison of the metabolic effects of recombinant human insulin-like growth factor-I and insulin: dose-response relationships in healthy young and middle-aged adults. J Clin Invest. 1994;93:1131-9.

7. Yakar S, Liu JL, Fernandes AM, Wu Y, Schally AV, Frystyk J, et al. Liver-specific IGF-I gene delection leads to muscle insulin insensitivity. Diabetes. 2001;50:1110-8.

8. Pereira AM, Biermasz NR, Roelfsema F, Romijn JA. Pharmacologic therapies for acromegaly: a review of their effects on glucose metabolism and insulin resistence. Treat Endocrinol. 2005; 4:43-53.

9. Melmed S. Acromegaly. N Engl J Med. 1990; 322:966-77.

10. Ayuk J, Clayton RN, Holtder G, Sheppard MC, Stewart PM, Bates AS. Growth hormone and pituitary radiotherapy, but not serum insulin-like growth factor-I concentrations, predict excess mortality in patients with acromegaly. J Clin Endocrinol Metab. 2004; 89:1613-7.

11. Colao A, Baldelli R, Marzullo P, Ferretti E, Ferono D, Gargiulo P, et al. Systemic hypertension and impaired glucose tolerance are independently correlated to the severity of the acromegalic cardiomyopathy. J Clin Endocrinol Metab. 2000; 85:193-9.

12. Holdaway IM, Rajassorya RC, Gamble GD. Factors influencing mortality in acromegaly. J Clin Endocrinol Metab. 2004; 89:667-74.

13. Tamburrano G, Durante C, Baldelli R. Therapy of diabetes and dyslipidemia in acromegaly. Pituitary. 2002; 5:27-31.

14. Tavares EF, Dalbosco IS, Abucham J, Russo EM. True insulin and intact proinsulin levels in acromegalic patients. Eur J Endocrinol. 1996; 134:549-53.

15. Fredstorp L, Werner S, Bang P, Hall K. Inverse correlation between insulin-like growth factor binding protein-1 and insulin in patients with acromegaly during treatment with somatostatin analogue octreotide. Clin Endocrinol. 1994; 41:495-501.

16. Ezzat S, Ren SG, Braunstein GD, Melmed S. Octreotide stimulates insulin-like growth factor-binding protein-1: a potential pituitary-independent mechanism for drug action. J Clin Endocrinol Metab. 1992; 75:1459-63.

17. Ezzat S, Ren SG, Braunstein GD, Melmed S. Octreotide stimulates insulin-like growth factor binding protein-1 (IGFBP-1) levels in acromegaly. J Clin Endocrinol Metab. 1991; 73:441-3.

18. Koop BL, Harris AG, Ezzat S. Effect of octreotide on glucose tolerance in acromegaly. Eur J Endocrinol. 1994; 130:581-6.

19. Elgin RG, Busby WH, Clemmons DR. An insulin-like growth factor (IGF) binding protein enhances the biologic response to IGF-I. Proc Natl Acad Sci USA. 1987; 84:3254-8.
20. Liu L, Brinkman A, Blat C, Harel L. IGFBP-1, an insulin-like growth factor binding protein, is a cell growth inibitor. Biochem Biophys Res Commun. 1991; 174:673-9.

21. Krejs GJ, Browne R, Raskin P. Effect of intravenous somatostatin on jejunal absorption of glucose, amino acids, water and eletrolytes. Gastroenterology. 1980; 78:26-31.

22. McKeage K, Cheer S, Wagstaff AJ. Octreotide Long-Acting Release (LAR) a review of its use in the management of acromegaly. Drugs. 2003; 63:2473-99.

23. Ipp BJ, Sinai Y, Bar-oz B. Somatostatin impairs clearance of exogenous insulin in humans. Diabetes. 1987; 36:673-7.

24. American Diabetes Association. Diagnosis and classification of diabetes mellitus. Diabetes Care. 2006; 29:S43-8.

25. Turner RC, Holman RR, Matthews D, Hockaday TDR, Peto J. Insulin deficiency and insulin resistance interaction in diabetes: estimation of their relative contribution by feedback analysis from basal plasma insulin and glucose concentrations. Metabolism. 1979; 28:1086-96.

26. Coutinho WF. Consenso latino- americano de obesidade. Arq Bras Endocrinol Metab. 1999; 43:21-67.

27. Donangelo I, Une K, Gadelha M. Diagnóstico e tratamento da acromegalia no Brasil. Arq Bras Endocrinol Metab. 2003; 47:331-46.

28. Musolino NRC, Knoepfelmacher M. Acromegalia e gigantismo. In: Coronho V, Petroianu A, Santana EM, Pimenta LG, editors. Tratado de endocrinologia e cirurgia endócrina, $1^{\underline{a}} \mathrm{ed}$. Rio de Janeiro: Guanabara Koogan AS; 2001. p.302-10.

29. Jallad RS, Musolino NRC, Salgado LR, Bronstein MD. Treatment of acromegaly with octreotide-LAR: extensive experience in a brazilian institution. Clin Endocrinol. 2005; 63:168-75.

30. Bengstsson BA, Brummer RJ, Edén S. Body composition in acromegaly. Clin Endocrinol. 1989; 31:481-90.

31. Baldelli R, Battista C, Leonetti F, Ghiggi MR, Ribaudo MC, PaoIoni $A$, et al. Glucose homeostasis in acromegaly: effects of long-acting somatostatin analogues treatment. Clin Endocrinol. 2003; 59:492-9.

32. Moller N, Schmitz O, Joorgensen JO, Astrup J, Back JF, Christensen SE, et al. Basal and insulin-stimulated substrate metabolism in patients with active acromegaly before and after adenomectomy. J Clin Endocrinol Metab. 1992; 74:1012-9.

33. Quabbe HJ, Plockinger U. Metabolic aspects of acromegaly and its treatment. Metabolism. 1996; 45:61-62.

34. Clemmons DR. Roles of insulin-like growth factor I and growth hormone in mediating insulin resistence in acromegaly. Pituitary. 2002; 5:181-3.

35. Hrebicek J, Janout V, Malincíková J, Horáková D, Cízek L. Detection of insulin resistance by simple quantitative insulin sensitivity check index QUICKI for epidemiological assessment and prevention. J Clin Endocrinol Metab. 2002; 87:144-7.

36. Damjanovic SS, Petakov MS, Raicevic S, Micic D, Marinkovic J, Dieguez C, et al. Serum leptin levels in patients with acromegaly before and after correction of hypersomatotropism by trans-sphenoidal surgery. J Clin Endocrinol Metab. 2000; 85:147-54.

37. Ronchi CL, Orsi E, Giavoli C, Cappiello V, Epaminonda P, BeckPeccoz $\mathrm{P}$, et al. Evaluation of insulin resistance in acromegalic patients before and after treatment with somatostatin analogues. J Endocrinol Invest. 2003; 26:533-8.

38. Ronchi C, Epaminonda P, Cappiello V, Beck-Peccoz P, Arosio M. Effects of two different somatostatin analogs on glucose tolerance in acromegaly. J Endocrinol Invest. 2002; 25:502-7. 
39. Parkinson C, Drake WM, Roberts ME, Meeran K, Besser GM, Trainer PJ. A comparison of the effects of pegvisomant and octreotide on glucose, insulin, gastrin, cholecystokinin, and pancreatic polypeptide responses to oral glucose and a standard mixed meal. J Clin Endocrinol Metab. 2002; 87:1797-1804.

40. Hirose T, Kuroda T, Otsuki M, Takagi M, Yamamoto H, Motomura $\mathrm{T}$, et al. A patient with acromegaly who showed remarkable improvement of hyperglycemia after treatment with octreotide. Intern Med. 1997; 36:345-50.

41. Cantalamessa L, Catania A, Baldini M, Orsatti A. Improvement of diabetes after treatment with somatostatin analogue SMS 201-995 in an acromegalic patient. Horm Metab Res. 1986; 18:790-1.

42. Ho KK, Jenkins AB, Furler SM, Borkman M, Chisholm DJ. Impact of octreotide, a long-acting somatostatin analogue, on glucose tolerance and insulin sensitivity in acromegaly. Clin Endocrinol. 1992; 36:271-9.
43. Vance ML, Harris AG. Long term treatment of 189 acromegalic patients with somatostatin analog octreotide. Arch Intern Med. 1991; 151:1573-8.

44. Arosio M, Macchelli S, Rossi CM, Casati G, Biella O, Faglia G. Effects of treatment with octreotide in acromegalic patients- a multicenter Italian study. Italian Multicenter Octreotide Study Group. Eur J Endocrinol. 1995; 133:430-9.

45. Colao A, Merola B, Ferone D, Lombardi G. Acromegaly. J Clin Endocrinol Metab. 1997; 82:2777-81.

\section{Endereço para correspondência:}

Mônica Roberto Gadelha

Rua Nascimento Silva, 555 ap. 101

22421-020 Rio de Janeiro, RJ

E-mail: mgadelha@hucff.ufr.br 